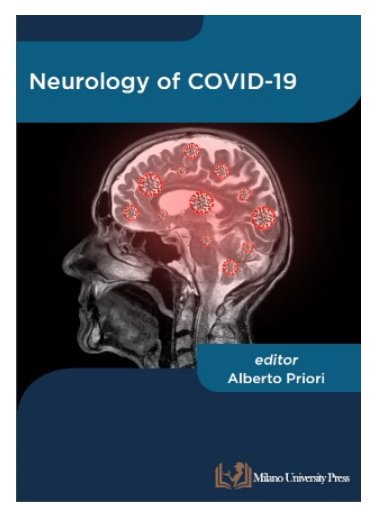

\title{
Neurology of COVID-19
}

Editor Alberto Priori

DOI: https://doi.org/10.54103/milanoup.57

Published by: Milano University Press

Via Festa del Perdono 7 - 20122 Milano

URL: https://milanoup.unimi.it/

E-mail: redazione.milanoup@unimi.it

\section{Chapter 18. Neuro-COVID in children}

DOI: https://doi.org/10.54103/milanoup.57.35

\section{List of Contributors}

\section{Ilaria Viganò}

Child Neuropsychiatry and Epilepsy Center, ASST Santi Paolo e Carlo, San Paolo University Hospital, Milan, Italy.

Email: ilaria.vigano@asst-santipaolocarlo.it

\section{Chiara Vannicola}

Child Neuropsychiatry and Epilepsy Center, ASST Santi Paolo e Carlo, Milan, Italy.

Email: chiara.vannicola@asst-santipaolocarlo.it

\section{Maria Paola Canevini}

Professor of Child Neurology

Epilepsy Center - Sleep Medicine Center, Childhood and Adolescence Neuropsychiatry Unit, ASST Santi Paolo e Carlo, San Paolo University Hospital, Milan, Italy;

Department of Health Sciences, University of Milan, Milan, Italy.

Email: mariapaola.canevini@unimi.it 


\title{
Chapter 18. Neuro-COVID in children
}

\author{
Ilaria Viganò , Chiara Vannicola*, Maria Paola Canevini \\ ${ }^{*}$ These authors equally contributed to this work
}

\section{Introduction}

Since the first cases identified in December 2019 in Wuhan, China, COVID-19 (COronaVIrus Disease 19), caused by severe acute respiratory syndrome coronavirus 2 (SARS-CoV-2), has rapidly progressed into a global pandemic. As of $17^{\text {th }}$ May 2021, there have been 162,704,139 confirmed cases of COVID-19 worldwide, including 3,374,052 deaths reported to the World Health Organization $(\mathrm{WHO})^{1}$.

Presentations of SARS-CoV-2 vary widely and range from asymptomatic to mild or moderate respiratory symptoms, to severe COVID-19 pneumonia, acute respiratory distress syndrome (ARDS), and multiorgan failure. Furthermore, many non-respiratory symptoms and manifestations have been reported, including variable long-lasting effects (the post-COVID syndrome or Long COVID) ${ }^{2,3}$.

COVID-19 may affect individuals of all ages, but the pediatric population seems to be less susceptible to SARS-CoV-2 infection. Moreover, reports of severe COVID-19 manifestations in children and adolescents are rare. The most common presenting manifestations of COVID-19 in children are non-specific symptoms, such as fever and cough; most children experienced asymptomatic, mild or moderate illness ${ }^{4,5}$. Furthermore, compared to adult populations, children were more likely to have normal leukocyte counts, while lymphopenia or lymphocytosis were infrequent ${ }^{5}$. Currently, few data regarding COVID-19 in the pediatric population are available and there has been no systematic description of the clinical spectrum of the disease in this group.

Although the pulmonary manifestations in COVID-19 are the most common, neurological symptoms are being recognized in an increasing number of patients, especially in severe disease. The first retrospective series published by Mao et al. reported neurological manifestations on 36\% of 214 patients hospitalized for COVID-19, including non-specific symptoms such as headache, myalgia, weakness, dizziness and specific neurological signs such as cerebrovascular disease, altered state of consciousness and encephalopathy ${ }^{6}$.

Neurological disease in children has already been reported in association with six of the seven human coronaviruses $(\mathrm{HCoV})$, including SARS-CoV-2, while the 
seventh, the Middle East respiratory syndrome coronavirus (MERS-CoV), has been described with neurological involvement in adults ${ }^{7-11}$. Neurological manifestations in children with $\mathrm{HCoV}$ are relatively rare, therefore there is little information about neurodevelopmental sequelae. However, cases of neurological disease associated with SARS-CoV-2 infections in children are rapidly emerging, suggesting a possible greater neurological involvement in the pediatric population affected by this virus than in other HCoVs. Only a small fraction of children affected by SARS-CoV-2 showed neurological manifestations such as seizures, encephalitis, stroke or neuropathies ${ }^{12}$. In most cases, severe neurological manifestations in the pediatric population appeared to be associated with the presence of Multisystem Inflammatory Syndrome (MIS-C). A review conducted by Chen in 2020 considering 187 children from the six latest reports of MIS-C cases showed that $34 \%$ suffered from neurological manifestations ${ }^{13-19}$. Of the 187 children studied, 64 had different neurological symptoms, most of which were headaches, positive meningeal signs (meningism), and altered mental status; taken together, most manifestations represent a central, rather than a peripheral involvement.

Neurological symptoms are thought to be secondary to a combination of different mechanisms, including the direct viral effect on the nervous system, the para- and post-infectious inflammation, and the neurological complications of the systemic effects of COVID-1920.

\section{Neurological manifestations of COVID-19 in children}

\section{Headache}

Headache is the most common neurological symptom related to COVID-19 and has been described as one of the presenting symptoms also in children ${ }^{5,21}$. From January $22^{\text {nd }}$ to May $30^{\text {th }} 2020$, according to case surveillance in the US, headache was reported in $15 \%$ of subjects in a cohort of 5,188 children with COVID-19 aged 0-9 years, and in 42\% of 12,689 children aged 10-19 years ${ }^{22}$. According to another recent review, headache, together with myalgia and fatigue, were predominant non-specific neurological manifestations, being reported in $16.7 \%$ of 3,707 patients aged 18 years ${ }^{12}$. Although headache is frequently reported by patients, this symptom appears to be in most cases non-specific or related to an inflammatory systemic process, fever or migraine exacerbation ${ }^{23,24}$. Headache could be secondary to meningeal irritation, encephalitis and encephalopathy only in a minority of patients. In these cases, headache is only one of a cluster of symptoms indicating an infectious and inflammatory process within the central nervous system (CNS), like seizures, impairment of consciousness, nausea and vomiting, neck stiffness, irritability, and others. Headache is also frequently reported in children with MIS-C, a recently recognized but uncommon Kawasaki syndrome-like hyperinflammatory childhood disorder related to SARS-CoV-2 
infection (see below). In a series of 58 hospitalized children from 8 different hospitals in England, headache was the most common symptom in MIS-C affecting $26 \%$ of patients ${ }^{25}$. Finally, headache could be one of the symptoms to persist after the resolution of the SARS-CoV-2 infection, also in children ${ }^{26}$.

\section{Seizures}

Over the past years, betacoronaviruses SARS-CoV and MERS-CoV have been associated with neuropathological alterations and neurological symptoms. The most common neurological manifestations of $\mathrm{HCoV}$ infections were seizures; in particular, simple febrile seizures.

Data about a possible neuro-invasiveness of SARS-CoV-2 as well as pathophysiological mechanisms give rise to the hypothesis that the infection could be associated with an increased risk of seizure recurrence or with the development of new onset and acute symptomatic seizures.

Up to now, there have been no important evidence in literature that SARSCoV-2 infection can cause worsening of the seizure in people with epilepsy. However, it is possible that the infection could favor the onset of seizures triggered by fever. Moreover, a severe disease course and advanced disease stage can result in hypoxic encephalopathy, cerebrovascular events, and the so-called "cytokine storm", which may trigger the development of acute seizures.

Different case reports regarding SARS-CoV-2 infections have suggested a neuro-invasive potential, with occurrence of neurological symptoms such as generalized tonic-clonic seizures, loss of consciousness, headache, and dizziness ${ }^{27}$.

Some case series reported the predominance of $\mathrm{HCoV}$-associated simple febrile seizures in children under one year of age ${ }^{28}$. In addition to febrile seizures, some cases of afebrile seizures associated to $\mathrm{HCoV}$ were reported. Esper et al. reported new-onset seizures in a patient affected by HCoV-HKU1. Central nervous system infections were ruled out; lumbar puncture, brain magnetic resonance imaging (MRI) were negative ${ }^{29}$. In 2020, Garcia-Howard et al. presented the case of a 3-month-old girl with a mildly symptomatic SARS-CoV-2 infection characterized by 2 days of fever and cough, which developed into two focal motor seizures with impaired awareness. Signs of meningo-encephalitis, other infections or active epilepsy were ruled out, and electroencephalogram and brain MRI were unremarkable. After 3 days, the child presented another afebrile motor seizure, and treatment with levetiracetam was started, with a favorable response. Whole exome sequencing was performed and revealed a pathogenic frameshift mutation in the PRRT2 gene in both the child and the mother, who had a history of late infantile febrile seizures ${ }^{30}$.

Moreover, 4 cases of status epilepticus associated with SARS-CoV-2 infection were described.

Swarz et al. described the case of a healthy 9-year-old child who tested positive for SARS-CoV-2, and who developed an acute-onset focal status 
epilepticus. EEG showed a continuous delta slowing throughout the right hemisphere without epileptiform features. Head CT and brain MRI were normal. After the acute event, the patient recovered without reported deficits ${ }^{31}$.

Another case of status epilepticus associated to encephalitis in a patient affected by SARS-CoV-2 was reported by McAbee et al. (see below) ${ }^{32}$.

Farley et al. described the case of status epilepticus in an 8-year old patient with SARS-CoV-2 infection who remained afebrile throughout the entire course of his illness; the patient had an underlying tic disorder without a previous history of epilepsy ${ }^{33}$. Saeed et al. reported the case of a previously healthy 3-year-old boy with SARS-CoV-2 infection who presented with repeated fever-induced seizure and status epilepticus. Brain CT scan revealed brain edema and 5 days later brain MRI showed intracerebral hemorrhage in the right occipital lobe ${ }^{34}$.

In all the cases reported, the patients had a complete recovery without neurological sequelae; however, long-term neurodevelopmental outcome is not yet evaluable due to short follow-up.

\section{Encephalitis}

Encephalitis is the inflammation of cerebral parenchyma caused by an infectious agent or aberrant reaction of the immune system against nervous system epitopes. For the diagnosis of infective encephalitis, the evidence of brain inflammation and the detection of the pathogen in cerebrospinal fluid (CSF) are necessary. Table 18.1 reports criteria for the diagnosis of SARS-CoV-2 encephalitis, according to the definition provided in a rapid review by Ellul et al. ${ }^{20}$.

Up to now, only a few reports of SARS-CoV-2 infection-associated encephalitis in children are available and virus has not been recovered from CSF in these few cases. McAbee et al. described a previously healthy 11-year-old boy who was admitted to the hospital with status epilepticus. The CSF analysis was compatible with a viral infection and the EEG registered frontal intermittent delta activity. The presence of SARS-CoV-2 was confirmed by nasopharyngeal swab; however, the detection of SARS-CoV-2 in the CSF is not reported. This case did not require specific treatment and the boy had a complete recovery in a few days ${ }^{32}$. Another case, described by Freij et al., was a previously healthy 5-year-old girl, presenting fever and severe headache for 7-10 days, subsequent confusion and seizure, and after another few days, lethargia and asymmetric pupils. Brain MRI showed an extensive meningoencephalitis involving cerebellum and corpus callosum with leptomeningeal enhancement over the surface of the brainstem and into the auditory canal. CSF was suggestive for viral encephalitis but negative for infection. The child died at day 32 of illness. The brain biopsy evidenced the presence of SARS-CoV-2 RNA and Mycobacterium tuberculosis complex DNA. In this case, the author hypothesized that the patient was asymptomatically infected with $M$. tuberculosis and the host immune system could not concurrently respond to the SARS-CoV-2 virus ${ }^{35}$. The pathophysiology of SARS-CoV-2-associated encephalitis is still not completely understood, but some mechanisms have been hypothesized. The dysregulation of 
the immune system, cell edema secondary to neuroinflammatory injury, immune-mediated neuronal damage due to "cytokine storm" syndrome, and the direct invasion of SARS-CoV-2 to the CNS could all be mechanisms contributing to the encephalitis process ${ }^{13}$.

Table 18.1: Diagnostic criteria for encephalitis associated with SARS-CoV-2 infection (Reproduced from ${ }^{20}$ with permission)

\section{SARS-CoV-2 meningitis, encephalitis, myelitis, or CNS vasculitis}

\section{Confirmed}

(1) SARS-CoV-2 detected in CSF or brain tissue or evidence of SARS-CoV-2-specific intrathecal antibody

(2) no other explanatory pathogen or cause found

\section{Probable}

(1) SARS-CoV-2 detected in respiratory or other non-CNS sample, or evidence of SARS$\mathrm{CoV}-2$-specific antibody in serum indicating acute infection

(2) no other explanatory pathogen or cause found

\section{Possible}

Patient meets suspected case definition of COVID-19 according to national or WHO guidance on the basis of clinical symptoms and epidemiological risk factors; in the context of known community SARS-CoV-2 transmission, supportive features include the following: the new onset of at least one of cough, fever, muscle aches, loss of smell, or loss of taste; lymphopenia or raised D-dimer level; and radiological evidence of abnormalities consistent with infection or inflammation (eg, ground glass changes)

In conclusion, a surprisingly small number of COVID-19 children develop classic encephalitic symptoms and data demonstrating SARS-CoV-2 in CSF are inconsistent. Clearly, more data are needed to clarify the potential role of SARS-CoV-2 in determining encephalitis.

\section{Stroke and cerebrovascular disease}

As regards cerebrovascular involvement, different reports of strokes and other types of cerebrovascular diseases in association with SARS-CoV-2 infections are available, whereas they were not previously described in association with other HCoVs. In Table 18.2 diagnostic criteria for stroke associated to SARS-CoV-2 infection are reported.

Regev et al. reported a case of a 16-year-old boy with unremarkable medical history who, after exposure to a COVID-19 patient, developed fever, sore throat, fatigue, abdominal pain, headache, and nuchal rigidity. After hospitalization, the patient developed septic shock requiring intubation. Diagnostic workup, including CSF and brain MRI documented the presence of a cerebrovascular disease involving 
microvascular structures, probably due to an inflammatory process. The patient recovered within 2 weeks after discharge, although muscular weakness persisted ${ }^{36}$.

Table 18.2: Diagnostic criteria for stroke associated with SARS-CoV-2 infection

(Reproduced from ${ }^{20}$ with permission)

\section{Stroke associated with SARS-CoV-2 infection}

\section{Probable association}

(1) Either SARS-CoV-2 detected in CSF or other sample, or evidence of SARS-CoV-2specific antibody in serum indicating acute infection;

(2) no other known traditional cardiovascular risk factors

\section{Possible association}

(1) Either SARS-CoV-2 detected in CSF or other sample, or evidence of SARS-CoV-2specific antibody indicating acute infection;

(2) other traditional cardiovascular risk factors

Schupper et al. described 2 cases of cerebrovascular events in a 2 -month-old infant and a 5-year-old child. The first had a medical history of tracheomalacia with tracheostomy and presented with respiratory failure requiring Extra Corporeal Membrane Oxygenation (ECMO). Head CT showed bilateral middle and posterior cerebral artery infarctions with hemorrhagic transformation, and brain MRI documented the presence of evolving hemorrhagic infarctions of bilateral occipitoparietal lobes, left temporal and frontal lobes; EEG showed non-convulsive status epilepticus. At time of publication, the patient was still on mechanical ventilation. The second case was a healthy 5-year-old child who was admitted to hospital after several days of fever, cough, and abdominal pain, with cardiogenic shock and cardiopulmonary failure requiring ECMO. Head CT showed right middle cerebral artery infarction, cerebral edema, and diffuse contralateral subarachnoid hemorrhage. After 5 days on ECMO, the child developed bilateral fixed and dilated pupils followed by loss of brainstem reflexes ${ }^{37}$. A similar case was reported by Kihira et al. describing a healthy 5 -year-old child who after 3 days of fever, cough and abdominal pain presented deterioration and cardiogenic shock requiring ECMO; the child died. A large right anterior and middle cerebral artery infarction with subarachnoid hemorrhage in the left hemisphere was documented by head CT ${ }^{38}$.

Another case of stroke in a patient with SARS-CoV-2 infection was reported by Tiwari et al. The patient, a 9-year-old child, presented with fever, frontal headache, vomiting and progressive right hemisome weakness that evolved to complete hemiplegia. CT angiography was performed and documented a multifocal smooth stenosis of both intracranial internal carotid arteries, right middle cerebral artery, and segments of the anterior cerebral arteries. The patient partially recovered and was on rehabilitation at time of publication ${ }^{39}$. Furthermore, Freij et al. and Essajee et al. reported 
cerebrovascular involvement in 2 patients with concurrence of active $M$. tuberculosis infection and SARS-CoV-2 infection. (See above for the case described by Freij et $\mathrm{al}^{35}$ ).

The case described by Essajee et al. was a healthy two and a half year old girl who presented with an acute onset lethargy, right mydriasis and ptosis, and left-sided hemiparesis. She also had progressively enlarging cervical lymphadenopathy and decreased appetite. Head CT showed pan-hydrocephalus, basal meningeal enhancement and infarction involving the anterior limb of the right internal capsule, lentiform nucleus, and thalamus. Cerebral sinus venous thrombosis was documented.

Both the authors postulated that the hyperinflammatory status caused by the overlapping infections resulted in endothelial damage, which exacerbated coagulopathy and stroke risk ${ }^{40}$. Two more case reports described focal cerebral arteriopathy involving the left middle cerebral artery with acute infarctions of the left insula and basal ganglia in children who tested positive for SARS-CoV-2 ${ }^{41,42}$. The most accredited hypothesis up to now, based on the evidence from adult patients, is that cerebrovascular events resulted from the SARS-CoV-2 "cytokine storm" causing inflammation-induced focal vasculitis.

\section{Acute disseminated encephalomyelitis}

Acute disseminated encephalomyelitis (ADEM) is a syndrome characterized by multifocal demyelination and presenting with focal neurological symptoms, typically occurring weeks after an infection. A provisional definition of ADEM related to SARS-CoV-2 infection is shown in Table 18.3.

Table 18.3: Diagnostic criteria for ADEM associated

with SARS-CoV-2 infection (Reproduced from ${ }^{20}$ with permission)

Acute disseminated encephalomyelitis associated with SARS-CoV-2 infection, GuillainBarré syndrome, and other acute neuropathies associated with SARS-CoV-2 infection

\section{Probable association}

(1) Neurological disease onset within 6 weeks of acute infection

(2) either SARS-CoV-2 RNA detected in any sample or antibody evidence of acute SARS-CoV-2 infection

(3) no evidence of other commonly associated causes

\section{Possible association}

(1) Neurological disease onset within 6 weeks of acute infection

(2) either SARS-CoV-2 RNA detected in any sample or antibody evidence of acute SARS-CoV-2 infection

(3) evidence of other commonly associated causes

ADEM has already been reported in association with infections by other HCoVs. In 2004, Ye et al. described the case of a 15-year-old boy who 
developed ADEM during HCoV-OC43 infection ${ }^{43}$. De Miranda HenriquesSouza et al. reported the case of a previously healthy 12 -year-old female who presented with skin rash lasting 6 days, and headache and fever for 1 day. After 5 days she presented acute, progressive, bilateral, symmetrical motor weakness with inability to stand, walk, or handle objects. On the second day of hospitalization, she developed respiratory failure requiring intubation, quadriparesis, and absence of deep tendon reflexes. CSF analysis was negative for infections but nasopharyngeal swab was positive for SARS-CoV-2; brain MRI revealed a neuroradiological pattern consistent with ADEM.

The patient underwent 5-day therapy with methylprednisolone, which was repeated due to little improvement of her weakness after first pulse therapy. More than two months after the onset, this patient had still not completely recovered and was still unable to sit without support, had persistent spastic quadriparesis with global hyperreflexia, and did not have complete sphincter control ${ }^{44}$.

Another recently reported case was a 17-month-old girl who presented with irritability, neck stiffness, right-sided nasolabial fold flattening, left upper extremity rigidity, right upper extremity paresis, lower limb hyper-reflexia, and truncal ataxia. During hospitalization, the patient developed autonomic instability and impairment of consciousness, and was moved to the intensive care unit (ICU). Brain MRI revealed multifocal hyperintense T2 fluid-attenuated inversion recovery signals in subcortical and periventricular white matter. CSF analysis was unrevealing but nasopharyngeal SARS-CoV-2 PCR was positive, as was serum antibody testing. This patient had a complete recovery in 2 months after high-dose methylprednisolone and intravenous immunoglobulin therapy followed by rehabilitation ${ }^{45}$. ADEM has been reported also in a 6-year-old patient with Fisher-Evans syndrome (autoimmune hemolytic anemia, immune thrombocytopenia and/or autoimmune neutropenia), who was given sirolimus and thalidomide. She had positive nasopharyngeal swab for SARS-CoV-2 but no symptoms suggestive of COVID-19. After 10 days, the patient presented a generalized tonic-clonic seizure with spontaneous resolution. A brain MRI showed a pattern consistent with ADEM. The patient received methylprednisolone and had a favorable clinical course ${ }^{46}$. These cases suggest that clinicians should consider ADEM when evaluating a child with encephalopathy and neurological signs together with a recent diagnosis of COVID-19.

\section{Guillain-Barrè syndrome and other neuropathies}

Guillain-Barré syndrome (GBS) is an acute polyradiculopathy clinically characterized by rapidly evolving ascending limb weakness, areflexia and sensory symptoms ${ }^{20}$. GBS has already been reported in children with $\mathrm{HCoV}$ infections. A case of acute flaccid paralysis was reported by Turgay et al., describing a case of a healthy 3-year-old child who presented with fever and cough, followed, after a few days, by dyspnea, inability to chew, swallow or speak, reduced muscular strength 
and absent deep tendon reflexes. The patient developed respiratory failure that required intubation. CSF pressure, glucose, and protein were normal, and blood, urine, and CSF cultures were negative. Electromyography and brain and spine MRI were normal. A nasopharyngeal swab revealed the presence of $\mathrm{HCoV}-229 \mathrm{E}$ and $\mathrm{HCoV}-\mathrm{OC} 43$ co-infection. Treatment with intravenous immunoglobulin was effective and after 2 weeks the patient could walk with support ${ }^{47}$.

Another case of acute flaccid paralysis associated with $\mathrm{HCoV}-\mathrm{OC} 43$ was reported by Sharma et al. The patient is a 5-year-old healthy male presenting with a left Bell palsy, reduced muscular strength, generalized hypotonia, inability to walk or to raise his arms above the shoulders, dysmetria, ataxia, dysphagia with drooling. CSF showed albumin-cytologic dissociation with no cells, and elevated CSF total protein, elevated albumin index (39.8; reference $\leq 9)$ and $\operatorname{Ig} G$ index (0.89; reference $\leq 0.66$ ), normal myelin basic protein, no oligoclonal bands. MRI showed brain and spine enhancement of the left bulbar nerve complex and anterior and posterior cervical nerve roots. Clinical and instrumental examinations suggested GBS diagnosis. The patient was treated with non-invasive ventilation for acute respiratory failure, and intravenous immunoglobulin, with a partial recovery after 2 weeks $^{48}$. Khalifa et al. reported a case of GBS associated with SARS-CoV-2 infection in a previously healthy 11-year-old male who, a month after upper respiratory infection and fever, developed acute symmetrical muscular weakness of the lower extremities and loss of deep tendon reflexes, tingling, impaired sensitivity. CSF and brain and spine MRI showing, respectively, albumin-cytologic dissociation and enhancement of the cauda equina nerve roots, in addition to evidence of a demyelinating process at nerve conduction studies, were consistent with a diagnosis of GBS. The patient was treated with intravenous immunoglobulin and after 2 weeks showed improved strength and sensory symptoms ${ }^{49}$. Gaur et al. described the case of a 3-year-old healthy child with asymptomatic SARS-CoV-2 infection, who developed transverse myelitis presenting with progressive extremity weakness resulting in flaccid tetraparesis, areflexia, and impaired sensitivity. Spine MRI showed swelling of cervical spinal cord with a lesion involving most of the transverse spinal cord from the lower medulla to the midthoracic level. CSF studies revealed pleocytosis and mildly elevated proteins ${ }^{50}$.

There is no direct evidence of a causative relationship between GBS or other acute flaccid paralysis and SARS-CoV-2 infection but the reports of similar cases and the data available about adult patients can raise the hypothesis that SARS-CoV-2 virus may be a possible trigger.

Other viruses such as Epstein-Barr virus (EBV), cytomegalovirus (CMV) and human immunodeficiency virus (HIV) had previously been implicated in the pathogenesis of GBS. The suggested mechanism is an autoimmune reaction secondary to molecular mimicry between the surface glycoproteins of the offending pathogen and the structures on peripheral nerves causing the antibodies to attack the nerves and cause neurological symptom. It is, 
therefore, possible to hypothesize that SARS-CoV-2 could be a trigger for a neurological process as the basis of $\mathrm{GBS}^{51,52}$.

Furthermore, before the recent pandemic, few cases of GBS secondary to coronavirus infections had been reported, whereas a recent systematic review documented a significant increase in the number of patients with GBS during the COVID-19 pandemic $^{53}$.

Table 18.4: Diagnostic criteria for MIS-C associated with SARS-CoV-2 infection according to Centers for Disease Control and Prevention definition (Reproduced from ${ }^{55}$ with permission)

\begin{tabular}{|l|}
\hline CDC case definition \\
\hline All 4 criteria must be met: \\
\hline 1. Age $<21$ years \\
\hline 2. Clinical presentation consistent with MIS-C, including all of the following: \\
- Fever: Documented fever $>38.0^{\circ} \mathrm{C}\left(100.4^{\circ} \mathrm{F}\right)$ for $\geq 24$ hours or Report of \\
- subjective fever lasting $\geq 24$ hours \\
- Laboratory evidence of inflammation, Including, but not limited to, any of the \\
following: \\
- Elevated CRP \\
- Elevated ESR \\
- Elevated fibrinogen \\
- Elevated procalcitonin \\
- Elevated D-dimer \\
- Elevated ferritin \\
- Elevated LDH \\
- Elevated IL-6 level \\
- Neutrophilia \\
- Lymphocytopenia \\
- Hypoalbuminemia \\
- Multisystem involvement (2 or more organ systems involved): \\
- Cardiovascular (eg, shock, elevated troponin, elevated BNP, abnormal \\
- echocardiogram, arrhythmia) \\
- Respiratory (eg, pneumonia, ARDS, pulmonary embolism) \\
- Neurologic (eg, seizure, stroke, aseptic meningitis) \\
- Hematologic (eg, coagulopathy) \\
- Gastrointestinal (eg, abdominal pain, vomiting, diarrhea, elevated liver enzymes, \\
- Dileus, gastrointestinal bleeding) \\
- Severe illness requiring hospitalization \\
\hline No alternative plausible diagnoses \\
\hline Recent or current SARS-CoV-2 infection or exposure (any of the following): \\
- Positive SARS-CoV-2 RT-PCR \\
- Positive serology antigen test \\
COVID-19 exposure within the 4 weeks prior to the onset of symptoms \\
\hline
\end{tabular}




\section{Neurological manifestations in Multisystem Inflamma- tory Syndrome in Children}

In contrast to most children with SARS-CoV-2 who develop only mild symptoms which usually do not require medical intervention, in April 2020, a group of clinicians from the UK described 8 previously healthy children presenting with fever, cardiovascular shock, and hyperinflammation ${ }^{54}$. In May 2020, the Centers for Disease Control and Prevention (CDC) defined the criteria for MIS-C and published a health advisory requesting health care practitioners to report suspected MIS-C cases to local, state, and territorial public health authorities. Criteria for MIS-C according to the CDC and the WHO definition are reported in Tables 18.4 and 18.5.

Table 18.5: Diagnostic criteria for MIS-C associated with SARS-CoV-2 infection according to World Health Organization definition ${ }^{56}$

\begin{tabular}{|l|}
\hline WHO case definition \\
\hline All 6 criteria must be met: \\
\hline Age 0 to 19 years \\
\hline Fever for $\geq 3$ days \\
\hline Clinical signs of multisystem involvement (at least 2 of the following): \\
- Rash, bilateral nonpurulent conjunctivitis, or mucocutaneous inflammation signs (oral, hands, or \\
- feet) \\
- Hypotension or shock \\
- echocardiac dysfunction, pericarditis, valvulitis, or coronary abnormalities (including \\
- Evidence of coagulopathy (prolonged PT or PTT; elevated D-dimer) \\
\hline Elevated markers of inflammation (eg, ESR, CRP, or procalcitonin) \\
\hline $\begin{array}{l}\text { No other obvious microbial cause of inflammation, including bacterial sepsis and staphylococcal/ } \\
\text { streptococcal toxic shock syndromes }\end{array}$ \\
\hline Evidence of SARS-CoV-2 infection. Any of the following: \\
- Positive SARS-CoV-2 RT-PCR \\
- Positive serology \\
- Positive antigen test \\
\hline
\end{tabular}

MIS-C is a Kawasaki-like syndrome with hyperinflammation presenting with acute hypotension and cardiogenic shock. This syndrome is thought to be a post-infection inflammatory phenomenon due to an immune reaction following asymptomatic or pauci-symptomatic COVID-19. Children can develop toxic shock-like symptoms, hypoxia-ischemia, and damage to the heart, kidneys, and other organs $s^{57,58}$. Neurological manifestations are frequently described in MIS-C. In New York, 31-47\% of affected children reported neurological symptoms like headache, altered mental status, and encephalopathy ${ }^{19}$. A recent systematic review including eight studies reported neurological symptoms in $25-50 \%$ of children with MIS-C ${ }^{59}$. A multicenter study on 
children with MIS-C in the US described neurological complications such as altered mental status, seizures and encephalitis in $5-11 \%$ of cases ${ }^{57}$. Headache was the most common symptom also in patients with MIS-C, affecting $26 \%$ of a series of 58 patients $^{25}$. In another series of 27 children with MIS-C, 4 patients developed encephalopathy, headache, dysarthria, dysphagia, weakness, ataxia, and peripheral neuropathy. In 2 patients, lumbar puncture was performed, and CSF was negative for SARS-CoV-2. Three patients showed diffuse slowing at EEG. For all children, brain MRI showed signal abnormalities of the splenium of the corpus callosum ${ }^{60}$. Another series of 21 children from France reported irritability in 57\% and "other neurological features" not further specified in $29 \%$ of children studied ${ }^{61}$. In a recent case series of inpatients aged $<21$ years with positive test for SARS-CoV-2 in 61 US hospitals, MIS-C was diagnosed in 36\% of patients and in 35\% of patients with neurological involvement. Twenty patients with MIS-C who developed life-threatening COVID-19-related neurological conditions had an MIS-C diagnosis. Among these, 8 had severe encephalopathy, 3 had ischemic or hemorrhagic stroke, 6 had acute CNS infection or ADEM, 2 had acute fulminant cerebral edema, and one developed $\mathrm{GBS}^{62}$. Patients with neurological manifestations in MIS-C could frequently present with symptoms such as altered mental status or headache, which suggest a CNS infection. However, in the reported cases, no demonstration of SARS-CoV-2 was evident. These data seem to indicate that neurological symptoms of MIS-C could be secondary to a post-infectious immune response. The over-reaction of monocytes, macrophages, and T cells which produces the "cytokine storm" syndrome may contribute to the neuronal damage, further aggravated by the release of interleukin-6 $(\mathrm{IL}-6)^{13}$. In conclusion, the impact of SARS-CoV-2 on the developing CNS, the immature immune system, and the neural-immune maturation have to be considered, and further data are needed to understand the pathophysiological characteristics of SARS-CoV-2 neurological involvement in MIS-C.

\section{Conclusions and clinical work-up}

Neurological manifestations associated with SARS-CoV-2 infection are rare in children but include a wide variety of central and peripheral insults. Most common symptoms are non-specific (headache, weakness), but severe manifestations such as encephalopathy, seizures, demyelinating disorders, and cerebrovascular events are possible. Furthermore, the pathophysiological mechanism at the basis of neurological manifestations is still under investigation, due to the relatively brief period of observation of the COVID-19 pandemic.

While for unspecific symptoms most cases require no further investigation, when symptoms suggesting a neurological involvement are present, other tests are needed. In particular, clinicians should be guided by signs observed during 
the neurological examinations and the symptoms reported by patients themselves. If altered mental status, meningism and neurological signs are detected, the clinical work-up should include brain MRI, EEG, and, if the suspicion of meningoencephalitis is still not clarified, a lumbar puncture with CSF analysis, including SARS-CoV-2 PCR is indicated. If weakness and paresis are present and GBS is suspected, an electromyography with electroneurography should be considered and a CSF examination showing albuminocytologic dissociation could be supportive for the diagnosis.

In conclusion, the diagnostic process should be personalized, based on the diagnostic hypothesis, and addressed by the symptoms reported and signs detected in patients.

\section{Therapeutic recommendation and prognosis}

The therapeutic approach in SARS-CoV-2 should follow guidelines for each suspected disorder.

Patients with headache usually benefit from commonly used analgesic drugs like non-steroidal anti-inflammatory drugs (NSAIDs), as no specific treatment options for COVID-19-related headache have been reported. When treating seizures, clinicians should always consider the interactions between anti-seizure medication and drugs used for COVID-19. Caution is needed in particular for carbamazepine, phenytoin, phenobarbital and primidone, enzyme inducers. A list of drug interactions is reported at the web page: https://www.covid19-druginteractions.org. ${ }^{63}$

Clinicians should be guided by conventional inclusion and exclusion criteria for stroke treatment such as systemic fibrinolysis and mechanical thrombectomy, even during SARS-CoV-2 infection.

In addition, the treatment of SARS-CoV-2 infection-related GBS and acute neuropathies does not differ from the conventional treatment, even if performing plasma exchange could present some organizational problems when patients are infectious ${ }^{64}$. According to a recent cohort study reporting on neurological symptoms in SARS-CoV-2 infection in hospitalized children and adolescents in the UK, immunomodulation was used as treatment in $86 \%$ of patients with ADEM, 80\% of patients with GBS, and in the only patient with limbic encephalitis. Admission to a pediatric ICU admission was necessary in $29 \%$ of patients with ADEM, $20 \%$ of patients with GBS, and $56 \%$ of patients with severe encephalopathy ${ }^{65}$.

Prognosis is frequently good but cases with neurological sequelae have been reported.

As regards short-term prognosis, disability was the outcome in 57\% of children with ADEM, 40\% of patients with GBS, 11\% of patients with severe encephalopathy in the previously cited cohort of hospitalized children; in these 
groups, no death was reported ${ }^{65}$. Data on long-term outcome of pediatric patients with COVID-19-related neurological involvement are still lacking and should be collected in such a way as to allow effects on cognition and development to be evaluated.

\section{Take home message}

- Neurological involvement in SARS-CoV-2 infection in children is rare but has to be considered when symptoms suggesting central or peripheral nervous system disease are present.

- The pathophysiology of neurological diseases in SARS-CoV-2 infection is still not completely clear but probably includes neuroinflammatory injury, a dysregulation of the immune system, immune-mediated neuronal damage due to "cytokine storm" syndrome, and the potential direct invasion of SARS-CoV-2 to the CNS.

- The most common symptom is headache, but cases of encephalopathy, seizures, demyelinating disorders, and cerebrovascular events during SARS-CoV-2 infection in children have been reported.

- Neurological symptoms are more frequent and could be serious in patients with MIS-C.

- Treatment should follow guidelines for each disorder. 


\section{References}

1. https://covid19.who.int

2. Carfi A, Bernabei R, Landi F. Gemelli Against COVID-19 Post-Acute Care Study Group. Persistent symptoms in patients after acute COVID-19. JAMA. 2020;324:603-605.

3. Osuchowski MF, Winkler MS, Skirecki T, et al. COVID-19: Pathophysiology of Acute Disease 1. The COVID-19 puzzle: deciphering pathophysiology and phenotypes of a new disease entity. Lancet. 2021;9(6):622-642.

4. Mantovani A, Rinaldi E, Zusi C, et al. Coronavirus disease 2019 (COVID-19) in children and/or adolescents: a meta-analysis. Pediatr Res. 2021;89(4):733-737.

5. Cui X, Zhao Z, Zhang T, et al. A systematic review and meta-analysis of children with coronavirus disease 2019 (COVID-19). J Med Virol. 2021;93(2):1057-1069.

6. Mao L, Jin H, Wang M, et al. Neurologic Manifestations of hospitalized patients With Coronavirus Disease 2019 in Wuhan, China. JAMA Neurology. 2020;77(6):683690 .

7. Desforges M, Favreau DJ, Brison A, et al. Human coronaviruses: respiratory pathogens revisited as infectious neuroinvasive, neurotropic, and neuro- virulent agents. In: Neuroviral Infections: RNA Viruses and Retroviruses. Boca Raton, FL: CRC Press; 2013.

8. Li YC, Bai WZ, Hashikawa T. The neuroinvasive potential of SARS-CoV2 may be at least partially responsible for the respiratory failure of COVID-19 patients. J Med Virol. 2020;92:552e555.

9. Barmore J. 5-year-old with rare complication becomes first Michigan child to die of COVID-19. The Detroit News, April 19, 2020. Accessed: April 30, 2020.

10. Molteni M. What does Covid-19 do to your brain? Available from: www.wired. com, April 15, 2020. Accessed: April 30, 2020.

11. Algahtani H, Subahi A, Shirah B. Neurological complications of Middle East respiratory syndrome coronavirus: a report of two cases and review of the literature. Case Rep Neurol Med. 2016;2016.3502683. doi:10.1155/2016/3502683.

12. Panda PK, Sharawat IK, Panda P, et al. Neurological Complications of SARSCoV-2 Infection in Children: A Systematic Review and Meta-Analysis. J Trop Pediatr. 2021;67(3):fmaa070. doi:10.1093/tropej/fmaa070.

13. Chen TH. Neurological involvement associated with COVID-19 infection in children. J Neurol Sci. 2020;418:117096.

14. Belhadjer Z, Meot M, Bajolle F, et al. Acute heart failure in multisystem inflammatory syndrome in children (MIS-C) in the context of global SARS-CoV-2 pandemic. Circulation. 2020;142:429-436.

15. Pouletty M, Borocco C, Ouldali N, et al. Paediatric multisystem inflammatory syndrome temporally associated with SARS-CoV-2 mimicking Kawasaki disease (Kawa-COVID-19): a multicentre cohort. Ann Rheum Dis. 2020;79(8):999-1006.

16. Toubiana J, Poirault C, Corsia A, et al. Kawasaki-like multisystem inflammatory syndrome in children during the covid-19 pandemic in Paris, France: prospective observational study, BMJ. 2020;369:m2094. 
17. Chiotos K, Bassiri H, Behrens EM, et al., Multisystem inflammatory syndrome in children during the COVID-19 pandemic: a case series. J Pediatric Infect Dis Soc. 2020;9(3):393-398.

18. Verdoni L, Mazza A, Gervasoni A, et al. An outbreak of severe Kawasaki-like disease at the Italian epicentre of the SARS-CoV-2 epidemic: an observational cohort study. Lancet. 2020;395(10239):1771-1778.

19. Dufort EM, Koumans EH, Chow EJ, et al. Multisystem inflammatory syndrome in children in New York state. N Engl J Med. 2020;383(4):347-358.

20. Ellul MA, Benjamin L, Singh B, et al. Neurological associations of COVID-19. Lancet Neurol. 2020;19:767-783.

21. Swann OV, Holden KA, Turtle L, et al. Clinical characteristics of children and young people admitted to hospital with covid-19 in United Kingdom: prospective multicentre observational cohort study. BMJ. 2020;370:m3249.

22. Stokes EK, Zambrano LD, Anderson KN, et al. Coronavirus Disease 2019 Case Surveillance - United States, January 22-May 30, 2020. MMWR Morb Mortal Wkly Rep. 2020;69(24):759.

23. Poncet-Megemont L, Paris,ì P, Tronchere A, et al. High Prevalence of Headaches During Covid-19 Infection: A Retrospective Cohort Study. Headache J Head Face Pain. 2020;60(10):2578-2582.

24. Stafstrom CE and Jantzie LL. COVID-19: Neurological Considerations in Neonates and Children. Children. 2020;7:133.

25. Whittaker E, Bamford A, Kenny J, et al. Clinical Characteristics of 58 Children With a Pediatric Inflammatory Multisystem Syndrome Temporally Associated With SARS-CoV-2. JAMA. 2020;324(3):259-269.

26. Buonsenso D, Munblit D, De Rose C, et al. Preliminary evidence on long COVID in children. Acta Paediatr. 2021;110(7):2208-2211.

27. Lau KK, Yu WC, Chu CM, et al. Possible central nervous system infection by SARS coronavirus. Emerg Infect Dis. 2004;10:342-344.

28. Singer TG, Evankovich KD, Fisher K, et al. Coronavirus Infections in the Nervous System of Children: A Scoping Review Making the Case for Long-Term Neurodevelopmental Surveillance. Pediatr Neurol. 2021;117:47-63.

29. Esper F, Weibel C, Ferguson D, et al. Coronavirus HKU1 infection in the United States. Emerg Infect Dis. 2006;12:775-779.

30. García-Howard M, Herranz-Aguirre M, Moreno-Galarraga L, et al. Case report: benign infantile seizures temporally associated with COVID-19. Front Pediatr. 2020;8:507.

31. Swarz JA, Daily S, Niemi E, et al. COVID-19 infection presenting as acute-onset focal status epilepticus. Pediatr Neurol. 2020;112:7.

32. McAbee GN, Brosgol Y, Pavlakis S, et al. Encephalitis associated with COVID-19 infection in an 11-year-old child. Pediatr Neurol. 2020;109:94.

33. Farley M, Zuberi J. COVID-19 precipitating status epilepticus in a pediatric patient. Am J Case Rep. 2020;21:e925776-1-e925776-4. 
34. Saeed A, Shorafa E. Status epilepticus as a first presentation of COVID-19 infection in a 3 years old boy; case report and review the literature. IDCases. 2020;22:e00942.

35. Freij BJ, Gebara BM, Tariq R, et al. Fatal central nervous system co-infection with SARS-CoV-2 and tuberculosis in a healthy child. BMC Pediatr. 2020;20:429.

36. Regev T, Antebi M, Eytan D, et al. Pediatric inflammatory multisystem syndrome with central nervous system involvement and hypocomplementemia following SARS-COV-2 infection. Pediatr Infect Dis J. 2020;39:E206-eE207.

37. Schupper AJ, Yaeger KA, Morgenstern PF. Neurological manifestations of pediatric multi-system inflammatory syndrome potentially associated with COVID-19. Childs Nerv Syst. 2020;36:1579e1580.

38. Kihira S, Morgenstern PF, Raynes $\mathrm{H}$, et al. Fatal cerebral infarct in a child with COVID-19. Pediatr Radiol. 2020;50:1479e1480.

39. Tiwari L, Shekhar S, Bansal A, et al. COVID-19 associated arterial ischaemic stroke and multisystem inflammatory syndrome in children: a case report. Lancet Child Adolesc Heal. 2021;5:88e90.

40. Essajee F, Solomons R, Goussard P, et al. Child with tuberculous meningitis and COVID-19 coinfection complicated by extensive cerebral sinus venous thrombosis. BMJ Case Rep. 2020;13:e238597.

41. Gulko E, Overby P, Ali S, et al. Vessel Wall Enhancement and Focal Cerebral Arteriopathy in a Pediatric Patient with Acute Infarct and COVID-19 Infection. AJNR Am J Neuroradiol. 2020;41(12):2348-2350.

42. Mirzaee SMM, Gonçalves FG, Mohammadifard M, et al. Focal Cerebral Arteriopathy in a Pediatric Patient with COVID-19. Radiology. 2020;297(2):E274-E275.

43. Yeh EA, Collins A, Cohen M, et al. Detection of coronavirus in the central nervous system of a child with acute disseminated encephalomyelitis. Pediatrics. 2004;113:e73ee76.

44. de Miranda Henriques-Souza AM, de Melo ACMG, de Aguiar Coelho Silva Madeiro B, et al. Acute disseminated encephalomyelitis in a COVID-19 pediatric patient. Neuroradiology. 2020;63:141e145.

45. McLendon LA, Rao CK, Da Hora CC, et al. Post-COVID-19 Acute Disseminated Encephalomyelitis in a 17-Month-Old. Pediatrics. 2021;147(6):e2020049678.

46. Manzo ML, Galati C, Gallo C, et al. ADEM post-Sars-CoV-2 infection in a pediatric patient with Fisher-Evans syndrome. Neurol Sci. 2021;12:1-4.

47. Turgay C, Emine T, Ozlem K, et al. A rare cause of acute flaccid paralysis: human coronaviruses. J Pediatr Neurosci. 2015;10:280.

48. Sharma K, Tengsupakul S, Sanchez O, et al. Guillain-Barre syndrome with unilateral peripheral facial and bulbar palsy in a child: a case report. SAGE Open Med Case Rep. 2019;7:2050313X19838750.

49. Khalifa M, Zakaria F, Ragab Y, et al. Guillain-Barre syndrome associated with severe acute respiratory syndrome coronavirus 2 detection and coronavirus disease 2019 in a child. J Pediatr Infect Dis Soc. 2020;9:510e513. 
50. Gaur P, Dixon L, Jones B, et al. COVID-19-associated cytotoxic lesions of the corpus callosum. AJNR Am J Neuroradiol. 2020;41:1905e1907.

51. Pritchard J. Guillain-Barré syndrome. Clin Med (Lond). 2010;10(4):399-401.

52. Yuki N, Hartung H-P. Guillain-Barré Syndrome. N Engl J Med. 2012;366(24):22942304.

53. Gittermann LMT, Feris SNV, von Oetinger Giacoman A. Relation Between COVID-19 and Guillain-Barré Syndrome in Adults: A Systematic Review. Neurologı́a (English Edition) 2020;35(9):646-654.

54. Riphagen S, Gomez X, Gonzalez-Martinez C. Hyperinflammatory shock in children during COVID-19 pandemic. Lancet. 2020;395:1607-1608.

55. Centers for Disease Control and Prevention Health Alert Network (HAN). Multisystem Inflammatory Syndrome in Children (MIS-C) Associated with Coronavirus Disease 2019 (COVID-19). 2020. Available at: https://emergency.cdc.gov/ han/2020/han00432.asp

56. World Health Organization. Multisystem inflammatory syndrome in children and adolescents with COVID-19: Scientific Brief. 2020. Available at: https:// www.who.int/publications-detail/multisystem-inflammatory-syndrome-in-children-and-adolescents-with-covid-19

57. 57 Feldstein LR, Rose EB, Horwitz SM, et al. Multisystem Inflammatory Syndrome in U.S. Children and Adolescents. N Engl J Med. 2020;383(4):334-346.

58. Nakra NA, Blumberg DA, Herrera-Guerra A, et al. Multi-System Inflammatory Syndrome in Children (MIS-C) Following SARS-CoV-2 Infection: Review of Clinical Presentation, Hypothetical Pathogenesis, and Proposed Management. Children. 2020;7:69.

59. Abrams JY, Godfred-Cato SE, Oster ME, et al. Multisystem Inflammatory Syndrome in Children (MIS-C) Associated with SARS-CoV-2: A Systematic Review. J Pediatr. 2020;5;226:45-54.e1.

60. Abdel-Mannan O, Eyre M, Löbel U, et al. Neurologic and Radiographic Findings Associated With COVID-19 Infection in Children. JAMA Neurol. 2020;77(11):1440-1445.

61. Toubiana J, Poirault C, Corsia A, et al. Kawasaki-like multisystem inflammatory syndrome in children during the covid-19 pandemic in Paris, France: Prospective observational study. BMJ. 2020;369:m2094.

62. LaRovere KL, Riggs BJ, Poussaint TY, et al. Neurologic Involvement in Children and Adolescents Hospitalized in the United States for COVID-19 or Multisystem Inflammatory Syndrome. JAMA Neurol. 2021;78(5):536-547.

63. https://www.covid19-druginteractions.org

64. Orsucci D, Ienco EC, Nocita G, et al. Neurological features of COVID-19 and their treatment: a review. Drugs Context. 2020;9:2020-5-1.

65. Ray STJ, Abdel-Mannan O, Sa M, et al. Neurological manifestations of SARSCoV-2 infection in hospitalised children and adolescents in the UK: a prospective national cohort study. Lancet Child Adolesc Health. 2021:S2352-4642(21)00193-0. 